\title{
ClusTrast: a short read de novo transcript isoform assembler guided by clustered contigs
}

\author{
Karl Johan Westrin, westrin@kth.se ${ }^{1}$ \\ Warren W. Kretzschmar, wk@warrenwk.com ${ }^{1,2}$ \\ Olof Emanuelsson, olofem@kth.se ${ }^{1}$
}

\begin{abstract}
${ }^{1}$ Science for Life Laboratory, Department of Gene Technology, KTH Royal Institute of Technology, SE-171 65, Solna, Sweden

${ }^{2}$ Center for Hematology and Regenerative Medicine (HERM), Department of Medicine Huddinge, Karolinska Institute, SE-141 52, Flemingsberg, Sweden
\end{abstract}

\begin{abstract}
Motivation: Transcriptome assembly from RNA-sequencing data in species without a reliable reference genome has to be performed de novo, but studies have shown that de novo methods often have inadequate reconstruction ability of transcript isoforms. This impedes the study of alternative splicing, in particular for lowly expressed isoforms.

Result: We present the de novo transcript isoform assembler ClusTrast, which clusters a set of guiding contigs by similarity, aligns short reads to the guiding contigs, and assembles each clustered set of short reads individually. We tested ClusTrast on datasets from six eukaryotic species, and showed that ClusTrast reconstructed more expressed known isoforms than any of the other tested de novo assemblers, at a moderate reduction in precision. An appreciable fraction were reconstructed to at least $95 \%$ of their length. We suggest that ClusTrast will be useful for studying alternative splicing in the absence of a reference genome.

Availability and implementation: The code and usage instructions are available at https://github.com/karljohanw/clustrast.

Contact: olofem@kth.se

Supplementary information: Supplementary material is available.
\end{abstract}

\section{Introduction}

In eukaryotes, many genes can produce RNA transcripts of differing base sequences called transcript isoforms. Transcript isoforms are created by alternative transcriptional start sites, splicing, or polyadenylation. Controlling transcript isoform expression allows a cell to regulate protein expression and thereby its behavior (Wang et al., 2008; Barbosa-Morais et al., 2012; Floor and Doudna, 2016). Changes in transcript isoform expression have been associated with developmental changes and tissue specificity in eukaryotes, and disease in humans (Fackenthal 
and Godley, 2008; Sterne-Weiler and Sanford, 2014; Xiong et al., 2015; Akhter et al., 2018). Thus, it is often important to clarify not only what genes that are expressed but also which transcript isoforms that are expressed.

The expression of genes and transcripts is often studied by RNA-sequencing, where short reads (SRs) derived from massively parallel shotgun sequencing are aligned to an organism's reference genome. With this approach, reconstructing transcripts is possible by using the reference genome as a guide (Garber et al., 2011). However, many non-model organisms do not have a high-quality reference genome available. In such cases, a commonly used approach is de novo assembly in which transcripts are assembled from the reads only. The assembled transcripts are sometimes referred to as contigs or reconstructed transcripts. Popular tools to perform de novo transcriptome assembly include Trans-ABySS (Robertson et al., 2010), Trinity (Grabherr et al., 2011), Oases (Schulz et al., 2012), and SOAP-denovo-Trans (Xie et al., 2014). An overview of current transcriptome assemblers is available, e.g., in Hölzer and Marz (2019).

In principle, these tools can also reconstruct transcript isoforms of the expressed genes, but in practice their sensitivity is poor. In Mus musculus, Schulz et al. (2012) reported that Oases, Trans-ABySS, and Trinity assembled 1.21, 1.25, and 1.01 transcripts per gene, respectively, whereas a reference-based assembler reconstructed 1.56 transcripts per gene. Bushmanova et al. (2019) also observed poor transcript isoform reconstruction performance of transcriptome assembly methods: while their method, rnaSPAdes, outperformed the other compared assemblers in gene reconstruction in Mus musculus, it assembled only 1.02 transcripts per gene. In the same comparison, Trinity managed to assemble the most transcripts, with a ratio of 1.11 transcripts per gene. The insufficient ability of current de novo transcriptome assemblers to reconstruct all expressed transcript isoforms of a gene was evident to us in our work on the DAL19 gene in spruce, Picea abies (Akhter et al., 2018): Only one out of four confirmed DAL19 transcript isoforms was reconstructed to at least $90 \%$ using Oases and two using Trinity. We performed a directed assembly that managed to reconstruct three of the four transcript isoforms, but this method did not scale to whole transcriptome assembly. These examples, and others, e.g. Hayer et al. (2015) and Thind et al. (2021), demonstrate that there is still much room for improvement in de novo transcript isoform assembly.

Another observation concerns the imperfect overlap between the sets of reconstructed transcripts from different de novo assembly tools. Smith-Unna et al. (2016) noted that out of Oases, Trinity, and SOAP-denovo-Trans, each assembler reconstructed a large number of bona fide transcripts that neither of the other assemblers managed to reconstruct. They concluded that combining assembly methods may be an effective way to improve the detection rate of transcripts.

We report the de novo transcriptome assembler ClusTrast, which builds upon our previous experience of transcript isoform assembly (Akhter et al., 2018). ClusTrast combines two assembly methods: Trans-ABySS and Shannon, and incorporates a novel approach to clustering and cluster-wise assembly of short reads. We assessed transcript isoform reconstruction performance of ClusTrast and several de novo transcriptome assemblers in six eukaryotic organisms and found that ClusTrast reconstructed more known transcript isoforms than any other assembler and reconstructed unknown (including misassembled) transcripts at a rate comparable to other assemblers. 
Table 1: Short read RNA-seq datasets accessed from the NCBI SRA database. RL=read length in bases. Species column, indicated in bold is the name to which the data set is referred to throughout this article. $\mathrm{RPS}_{\mathrm{S}}=$ million read pairs, before pre-processing (on the left) and after pre-processing (on the right).

\begin{tabular}{l||r|ll|rr} 
SRA ID & \multicolumn{1}{c|}{ RL } & Species & \multicolumn{2}{|c}{ RPs } \\
\hline \hline SRR1153470 & $2 \times 101$ & Human & Homo sapiens & 115 & 103 \\
SRR8632985 & $2 \times 76$ & Mouse & Mus musculus & 31 & 30 \\
SRR11341576 & $2 \times 150$ & Rice & Oryza satvia & 24 & 23 \\
SRR5344669.1 & $2 \times 130$ & Arabidopsis & Arabidopsis thaliana & 61 & 59 \\
SRR10728575 & $2 \times 150$ & Zebrafish & Danio rerio & 21 & 20 \\
SRR10853135 & $2 \times 150$ & Poplar & Populus trichocarpa & 31.9 & 31.7
\end{tabular}

\section{Materials and Methods}

\subsection{Datasets and annotations}

\subsubsection{Short read RNA-seq datasets for assembly generation}

We tested the de novo transcriptome assemblers using the NCBI SRA datasets in Table 1. They are all paired-end short read (SR) RNA-seq datasets. We pre-processed the datasets with fastp (Chen et al., 2018) with default parameters, which means removal of any remaining adapter sequences, quality pruning ( $\max 40 \%$ of the bases are allowed to have base quality $<16$, and at most five Ns per read), and exclusion of reads that ended up shorter than 15bp (see the supplementary material for details).

\subsubsection{Reference data sets for assembly evaluation}

We downloaded reference genome sequences as well as reference transcript annotations from Ensembl for each of the six species. We used the GTF file annotations of genes and transcripts, not including the abinitio annotations. We estimated the expression of all reference transcripts in each of the six datasets using RSEM (Li and Dewey, 2011) and defined a transcript isoform as expressed if the transcripts per million (TPM) reported by RSEM was greater than zero. Versions and commands for RSEM are listed in the supplementary material. We defined a gene as expressed if at least one of the transcript isoforms associated with that gene was expressed. The versions of the annotations used and the number of genes and isoforms we detected in each dataset are shown in Table 2.

\subsection{Transcriptome assembly generation}

We assembled the transcriptomes for all six datasets (Table 1) using Trans-ABySS (Robertson et al., 2010), Trinity (Grabherr et al., 2011), Oases (Schulz et al., 2012), SOAP-denovoTrans (Xie et al., 2014), BinPacker (Liu et al., 2016), Shannon (Kannan et al., 2016), rnaSPAdes (Bushmanova et al., 2019), TransLiG (Liu et al., 2019), RNA-Bloom (Nip et al., 2020), and ClusTrast. We used each assembler's own default parameters. Trans-ABySS and Oases can be run in a "multi- $k$ " mode where the assembler is first run with a single $k$-mer ("single- $k$ " mode; where a $k$-mer is a substring, with fixed length $k$, of a read) for several different $k$-mers and the resulting assemblies are merged into a single assembly. We used both the single- $k$ 
Table 2: Reference transcriptome sequence, genome sequence and annotation versions accessed from Ensembl (https://www.ensembl.org/index.html and https://plants.ensembl.org/index.html for non-plant and plant species, respectively), where the Version id suffix shows the Ensembl version. The number of genes and isoforms are counted from the reference transcriptome. Genes and isoforms are considered expressed if TPM $>0$ as calculated by RSEM on the datasets in Table 1.

\begin{tabular}{l|l||rr|rr}
\multicolumn{2}{c||}{ Reference } & \multicolumn{2}{c|}{ Total } & \multicolumn{2}{c}{ Expressed } \\
Species & Version id & Genes & Isoforms & Genes & Isoforms \\
\hline \hline Human & GRCh38.99 & 40491 & 190432 & 22510 & 102552 \\
Mouse & GRCm38.99 & 36711 & 119353 & 17400 & 52845 \\
Arabidopsis & TAIR10.48 & 27655 & 48359 & 23085 & 38004 \\
Rice & IRGSP-1.0.48 & 37967 & 44761 & 29703 & 34956 \\
Zebrafish & GRCz11.99 & 30628 & 57775 & 23963 & 35189 \\
Poplar & Pop_tri_v3.46 & 41335 & 73012 & 29400 & 44652
\end{tabular}

and multi- $k$ strategies for these two assemblers. We append $-\mathbf{M}$ to the name of a method if it uses a multi- $k$ strategy, and $\mathbf{- S}$ if it uses a single- $k$ strategy. Oases- $\mathbf{M}$ uses by default all odd $k$-mers from 19 to 31, but it only finished within less than 58 hours on the mouse, poplar, and arabidopsis datasets. On the rice dataset, it finished after $\sim 400$ hours. Therefore, for human and zebrafish, we used only Oases-S with $k=31$. The program versions and the executed commands are listed in the supplementary material.

We also generated a concatenated assembly from the Trans-ABySS and Shannon transcriptomes, referred to as $\operatorname{Tr} \mathrm{AB}+\mathrm{Sh}$, to examine if the clustering approach of ClusTrast improves the assembly quality.

\subsection{Transcriptome assembly evaluation}

To benchmark the assemblers by estimating precision (positive predicted value, PPV) and recall (sensitivity or true positive rate, TPR), we used the reference based transcriptome comparison tools SQANTI (Tardaguila et al., 2018) and Conditional Reciprocal Best BLAST (CRBB) (Aubry et al., 2014) as implemented in the TransRate package (Smith-Unna et al., 2016). Versions and commands for these tools can be found in the supplementary material. We only used reference transcripts that were considered expressed (2.1.2). All assembled transcripts were considered expressed, since they were reconstructed from actual RNA-seq data.

\subsubsection{Using SQANTI in evaluation}

We used SQANTI (Structural and Quality Annotation of Novel Transcript Isoforms) (Tardaguila et al., 2018) to classify assembled transcripts according to their splice junction matches with reference genes and transcript isoforms. When an assembled transcript is anti-sense to an annotated gene, SQANTI will classify that transcript as anti-sense. We extracted all transcripts classified as anti-sense, reverse-complemented them, and then reclassified them with SQANTI.

When an assembled transcript and a reference isoform have the same number of exons and same splice junctions, then SQANTI classifies it as a full splice match (FSM). When the assembled transcript has fewer exons than the reference but the splice junctions in the assembled transcript all exist in the reference, it is classified as an incomplete splice match (ISM). 
SQANTI assigns an ISM-classified assembled transcript to the shortest reference isoform that is consistent with the observed splice matches in the assembled transcript. SQANTI requires that an assembled transcript ends at a known splice junction in order to classify the assembled transcript as an ISM. Assembled transcripts classified by SQANTI as novel in catalog (NIC, when the splice junctions are known but there is a novel combination) and novel not in catalog (NNIC, with novel splice junctions) were not classified as true positives.

For recall, we counted all expressed reference isoforms with at least one assembled transcript that SQANTI classified as FSM or ISM (and with a certain fraction, 1.0-0.25, of the exons covered) as a true positive, and divided the total number of true positives by the total number of expressed reference isoforms. For precision, we counted each assembled isoform classified by SQANTI as FSM or ISM and covering at least a certain fraction (from 1.0 to 0.25 ) of the reference exons as a true positive, and divided the total number of true positives by the total number of assembled isoforms.

\subsubsection{Using CRBB in evaluation}

We used CRBB (Conditional Reciprocal Best BLAST) (Aubry et al., 2014) to classify assembled transcripts according to their similarity to reference transcripts. To this end, we used TransRate (Smith-Unna et al., 2016), which in turn used BLAST (Altschul et al., 1990) to align each assembled transcript to the set of reference transcripts, and each reference transcript to the set of assembled transcripts. A transcript is a CRBB hit when the transcript is the top hit in both BLAST alignments. We defined recall as the proportion of reference transcripts that have a CRBB hit covering the reference transcript to at least $100 \%-25 \%$. We defined precision as the proportion of assembled transcripts that are a CRBB hit covering the reference isoform to at least $100 \%-25 \%$.

\section{Results}

\subsection{ClusTrast method}

We developed a method for transcriptome assembly from short reads called ClusTrast.

\subsubsection{Overview}

Figure 1 shows a flowchart of the ClusTrast method. The only required input to ClusTrast is a file with short RNA-seq reads, referred to as SRs (short reads). Supplementary Figure S.1 illustrates an example of how the method works.

\subsubsection{Primary assembly and guiding contigs (GCs)}

In ClusTrast, Trans-ABySS (Robertson et al., 2010) is employed to create a "primary assembly" from the short reads. The primary assembly will by default be used as the set of guiding contigs (GCs) in ClusTrast. The guiding contigs are used in the next step to cluster the SRs (3.1.3). Guiding contigs may also be provided separately by the user, and could then serve as primary assembly if desired. The primary assembly is by default also merged into the final set of assembled transcripts (3.1.5), in order to capture isoforms that the cluster-wise assemblies (3.1.4) might fail to assemble due to low coverage. 


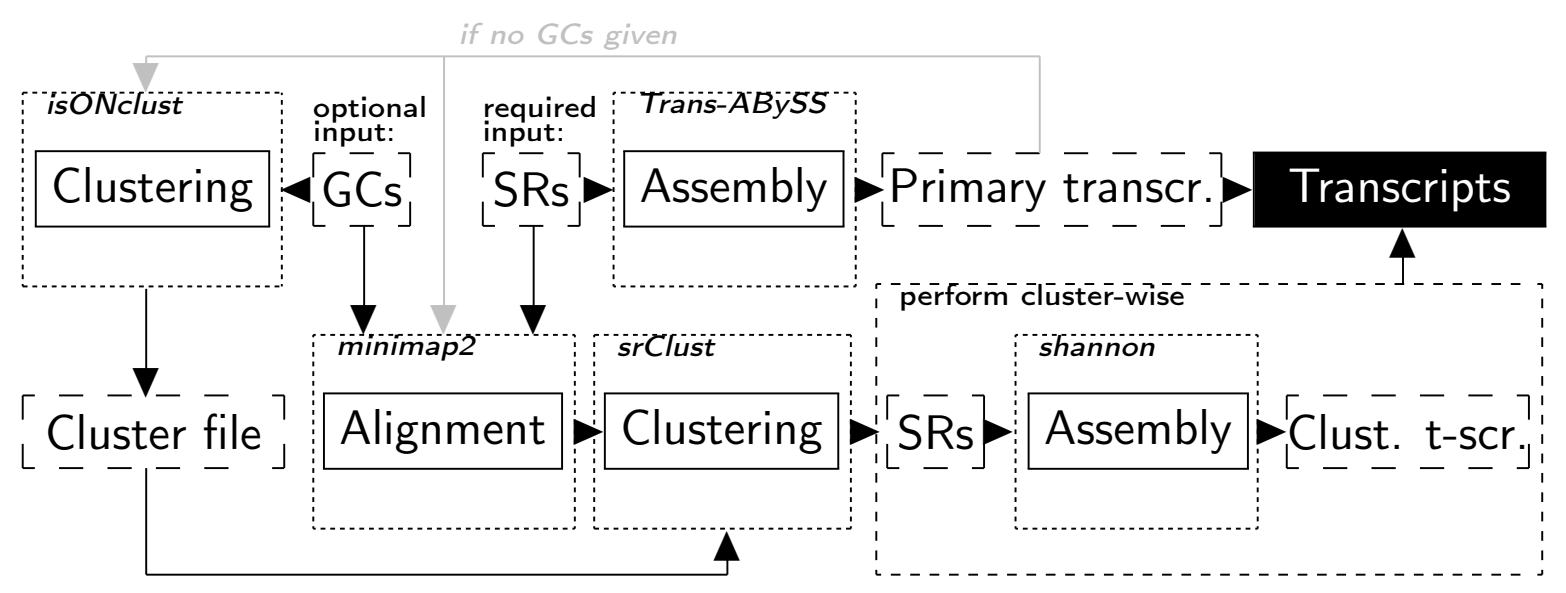

Figure 1: The ClusTrast pipeline. $\mathrm{SRs}=$ short reads, GCs = guiding contigs. The only required input data is the set of SRs from an RNA-seq experiment.

The original version of Trans-ABySS used several different $k$-mers and merged the resulting assemblies, in order to get both recall from small values of $k$ and precision from high values of $k$. Since a single- $k$ run uses much less memory (or is substantially faster) than a multi- $k$ run, we tried both strategies with ClusTrast. In this report, we have appended - $\mathbf{M}$ to the name of a method if it used a multi- $k$ strategy.

\subsubsection{Clustering of (a) GCs and (b) SRs}

(a) The clustering of the guiding contigs is performed with isONclust (Sahlin and Medvedev, 2020), a tool originally developed for clustering of PacBio CCS reads or ONT reads into gene families.

(b) The SRs are aligned to the guiding contigs with minimap2 (Li, 2018), using the preset option $-\mathrm{x}$ sr, intended for SR alignment, but included secondary alignments (can optionally be excluded in ClusTrast). Next, the SRs are assigned to the guiding contig clusters based on the alignment results. If a SR $x$ is aligned to guiding contigs $X_{1}$ and $X_{2}$, and $X_{1}$ belongs to cluster $n_{1}$ and $X_{2}$ belongs to cluster $n_{2}, x$ will be included in $n_{1}$ and $n_{2}$. Thus, the SRs have now been clustered.

\subsubsection{Clusterwise assembly}

The cluster-wise assembly in ClusTrast is performed by the transcriptome assembler Shannon (Kannan et al., 2016), and aims to be information theoretically optimal. Kannan et al. claim that Shannon can finish in linear time given $(i)$ diversity of transcript abundance and (ii) no loops in the graph, but do not address how it will deal with datasets not meeting these criteria. However, dividing the reads in the SR dataset into clusters before assembly will reduce the complexity of each individual assembly and lower the risk of violating these requirements. Because of this, and its aim to reconstruct as many transcripts as possible, Shannon is used for the cluster-wise assemblies in ClusTrast.

\subsubsection{Merging the assemblies}

The final step of ClusTrast is to merge the cluster-wise assemblies with the primary assembly by concatenation. Duplicate instances of the reconstructed transcripts are by default removed. 


\subsection{Transcriptome assembly evaluation}

Transcriptome assemblies for all compared assemblers, including ClusTrast, were generated as described in 2.2. Basic statistics of all assembled transcriptomes are available in Supplementary Table S.1-S.6.

\subsubsection{Evaluation with SQANTI}

We investigated how recall and precision changed when we varied the proportion of exons that an assembled transcript needs to recover in order to be considered a true positive. As this proportion was relaxed for the ISM classifications from 1.0 to 0.25 (for the FSM category it is by definition 1.0), the recall (Figure 2) and precision (Figure 3) increased. ClusTrast-M had the highest SQANTI recall of any assembler for all of the six datasets over the entire range. The assembler with the highest precision varied across datasets; it was ClusTrast-M in human and rice, Oases- $\mathbf{M}$ in mouse and poplar, rnaSPAdes in arabidopsis, and TransLiG in zebrafish.

Fixing the proportion at 0.5 (i.e., at least $50 \%$ of exons recovered for ISM), ClusTrast$\mathbf{M}$ detected more transcript isoforms than the established assemblers Trinity (1.3-2.5 fold increase), Oases-S (1.4-2.9 fold increase), and Trans-ABySS-M (1.1-1.4 fold increase) (Figure S.2 and Table S.7). Precision was comparable to Trinity (0.9-1.9 fold change), Oases-S (0.64-1.5 fold change) and Trans-ABySS-M (0.73-1.53 fold change).

To determine if the assemblers differed in how well they recovered isoforms of genes with more than one annotated isoform (alternative splicing), we calculated SQANTI recall of isoforms binned by genes according to the number of isoforms these genes expressed (Figure 4). In most cases the ranking of assemblers by recall did not change with increasing number of expressed isoforms per gene. ClusTrast-M came out on top over almost the entire range for 5 out of 6 datasets (although for mouse it was tied with $\operatorname{Tr} \mathrm{AB}+\mathrm{Sh}$ and for poplar it was tied with Oases-M and TrAB+Sh). The one exception was in arabidopsis where Oases-M, Trinity, and RNA-Bloom had a slower decrease in recall with isoforms per gene than the other assemblers.

We binned reference transcripts by expression quantile as measured by RSEM and observed that the SQANTI recall for ClusTrast-M was higher than all other assemblers across expression quantiles, except in arabidopsis where Oases-M had the highest recall in the 0-50th percentiles (Supplementary Figure S.3).

\subsubsection{Evaluation with CRBB}

We investigated CRBB recall and precision over the same proportion of required recovered exons as for SQANTI, 1.0-0.25, and observed an increase in recall and precision as this proportion was decreased. We observed some changes in the relative ordering of assemblers as shown in Figure 5 and Figure 6. In particular, rnaSPAdes performed comparably worse in the lower end.

Fixing the proportion at 0.5 , CRBB recall was higher for ClusTrast-M than for Trinity (1.1-1.7 fold increase) across all datasets, but not compared to all assemblers (Supplementary Figure S.4 and Supplementary Table S.8). ClusTrast-M performed the best on mouse, rice, and zebrafish, and least well on arabidopsis where it ranked fourth. In all datasets except arabidopsis and poplar we observed a trade-off between CRBB recall and precision. ClusTrast$\mathbf{M}$ clearly underperformed compared with Trinity with regard to CRBB precision $(0.2-0.67$ 
(a) Human

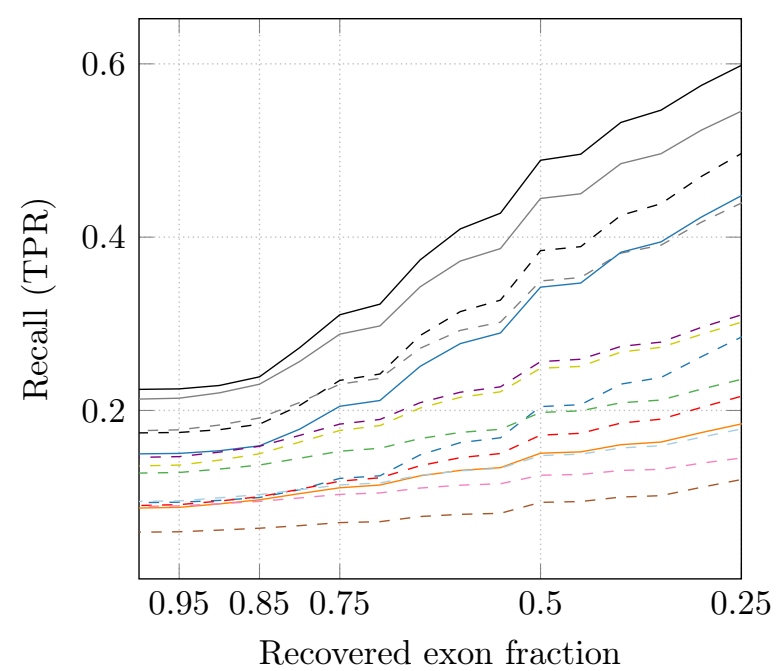

(c) Rice

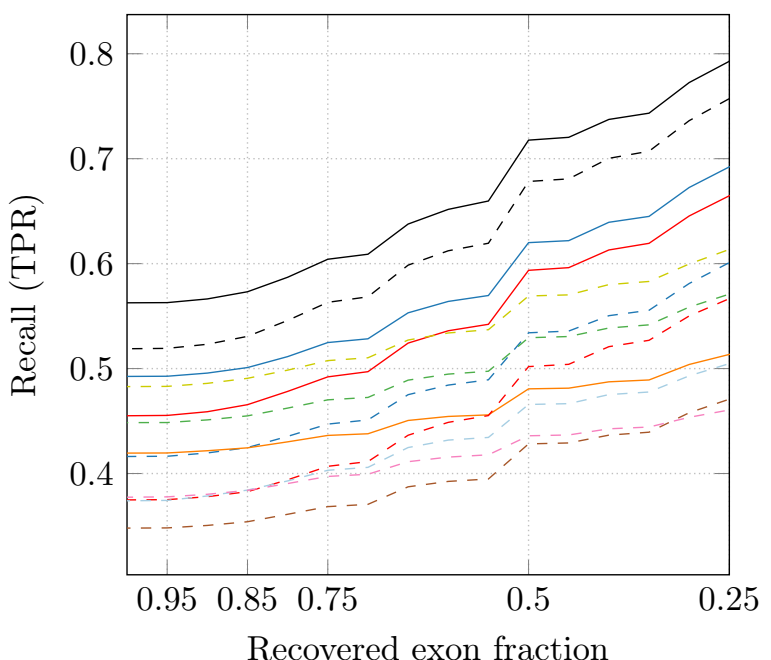

(e) Zebrafish

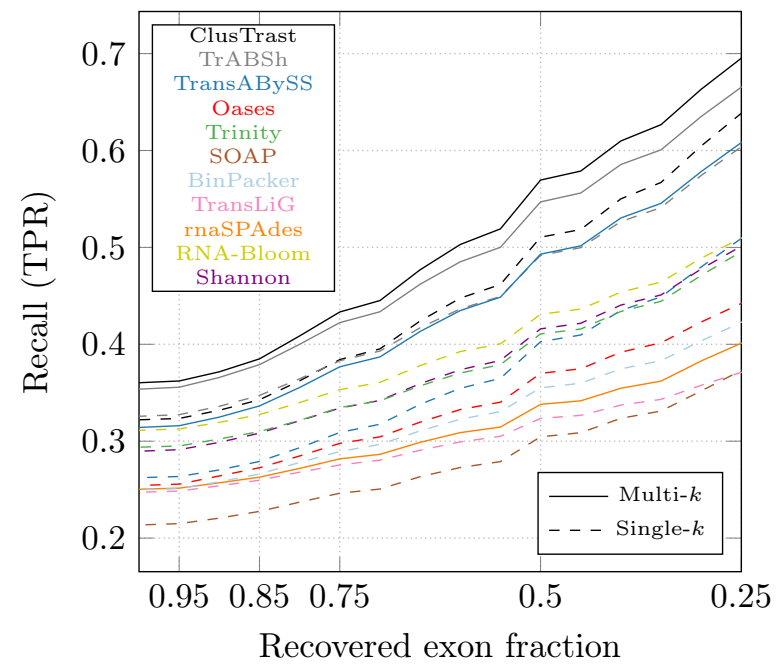

(b) Mouse

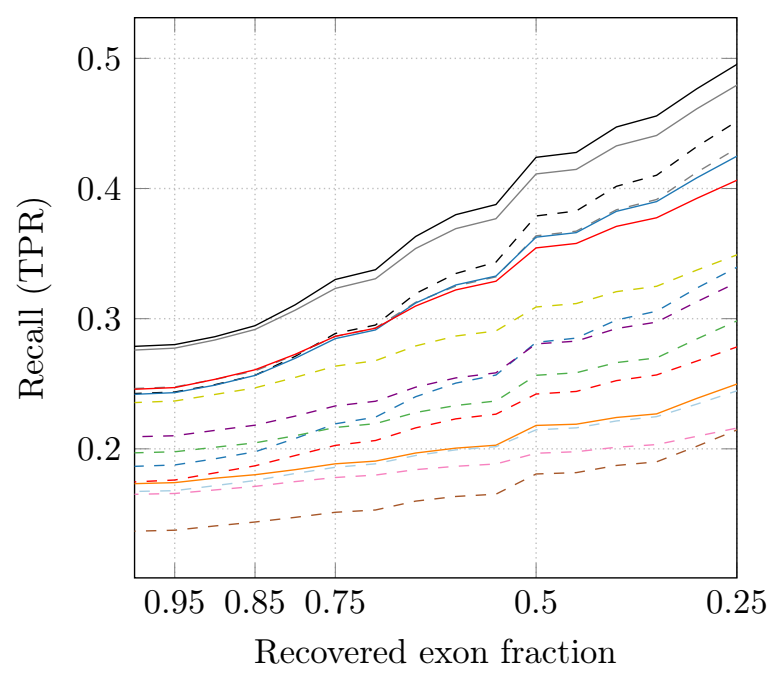

(d) Arabidopsis

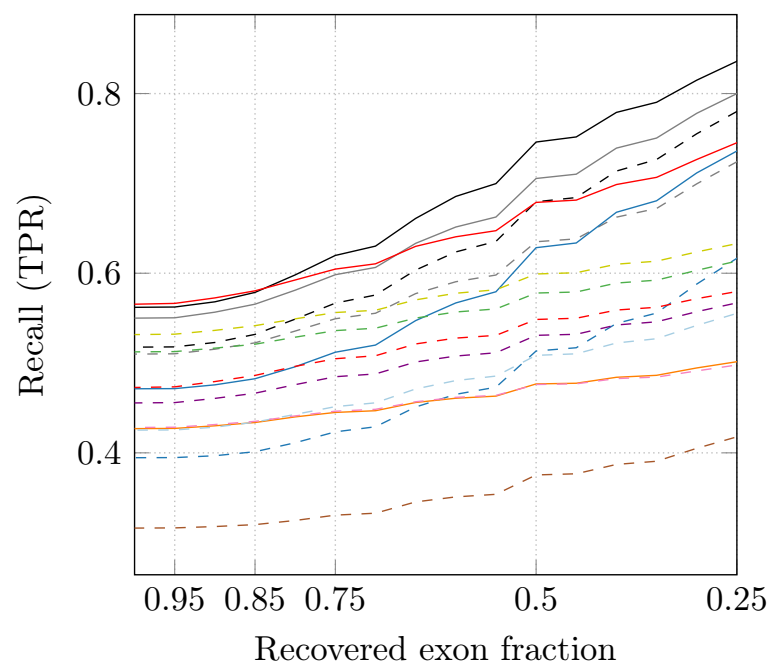

(f) Poplar

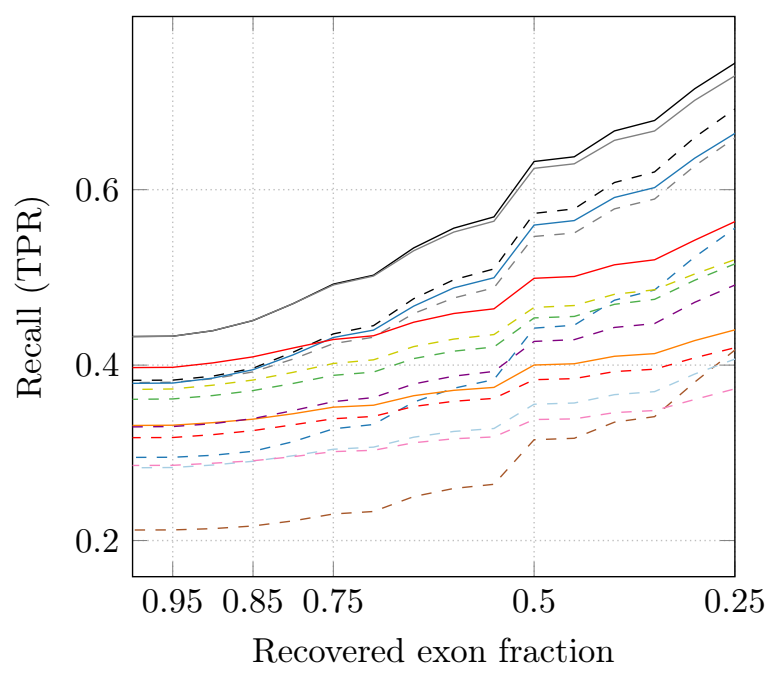

Figure 2: Proportion of reference isoforms with at least one SQANTI classification of FSM or ISM vs. the cumulative proportion of exons recovered by the assembly. 
(a) Human

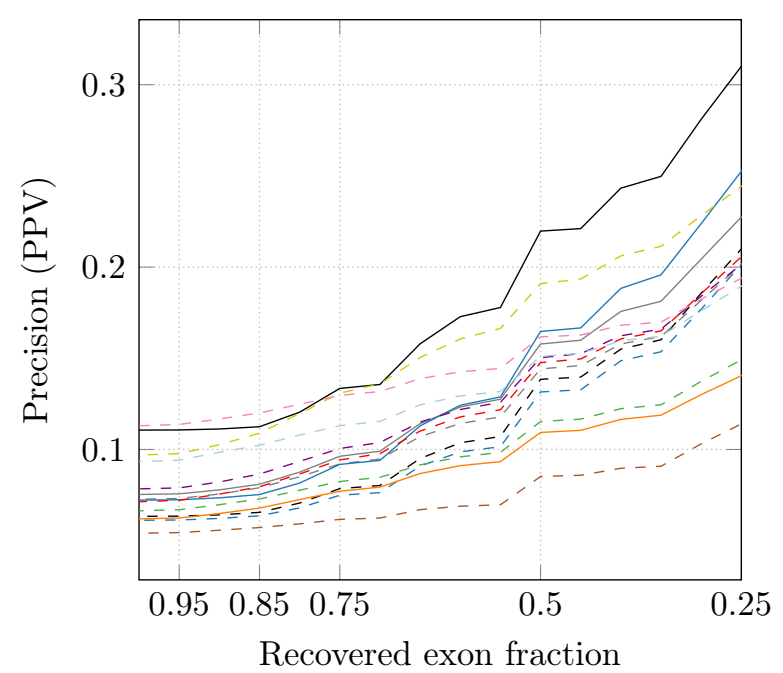

(c) Rice

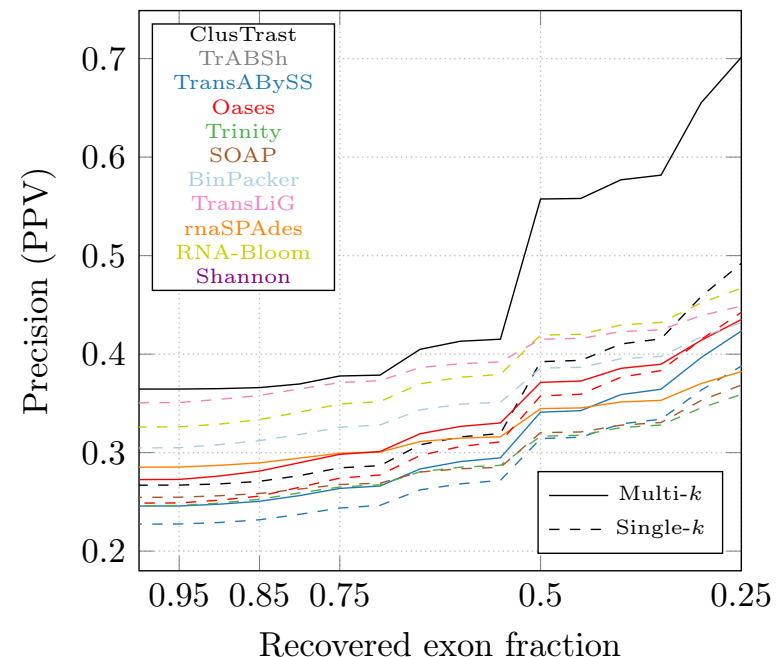

(e) Zebrafish

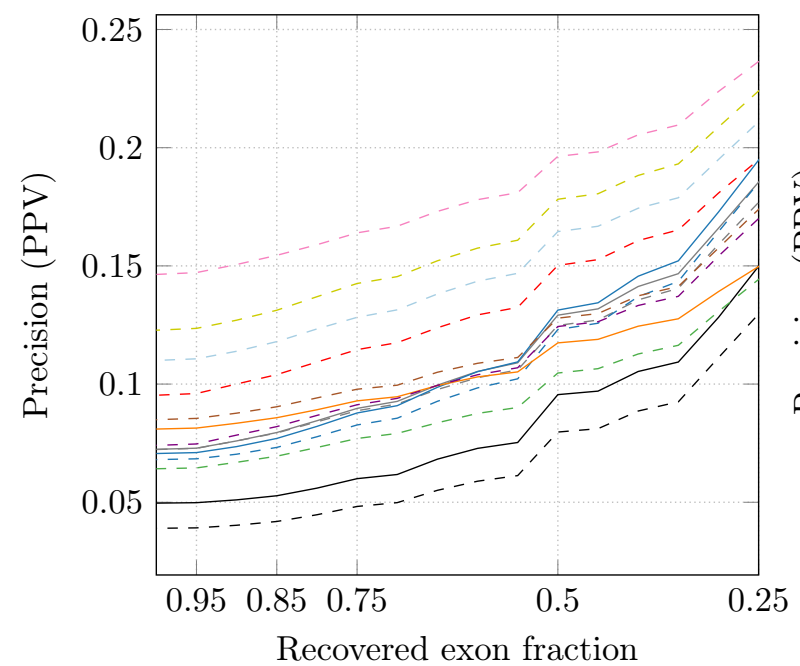

(b) Mouse

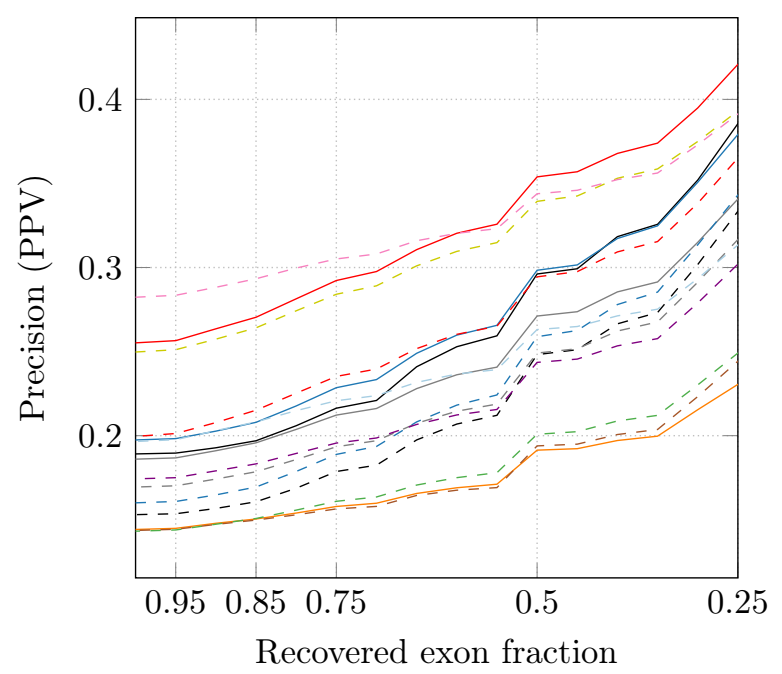

(d) Arabidopsis

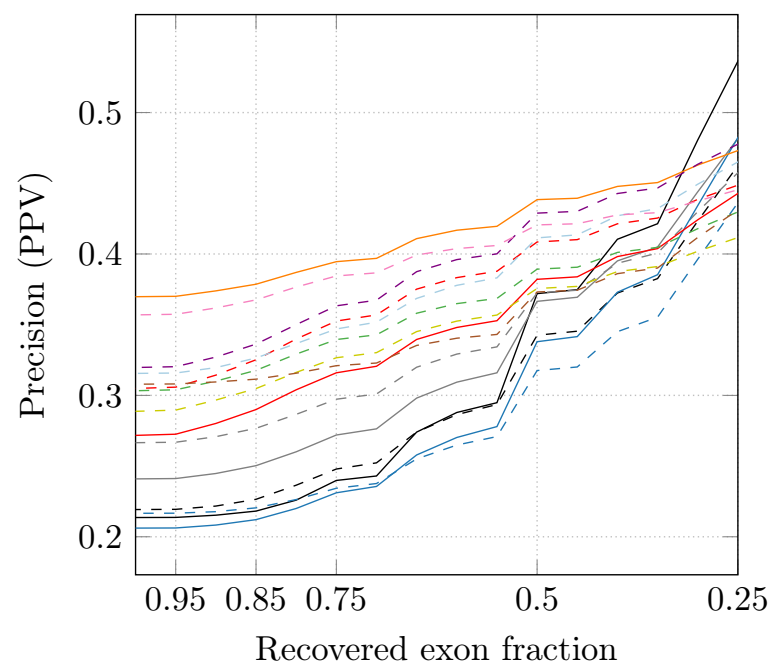

(f) Poplar

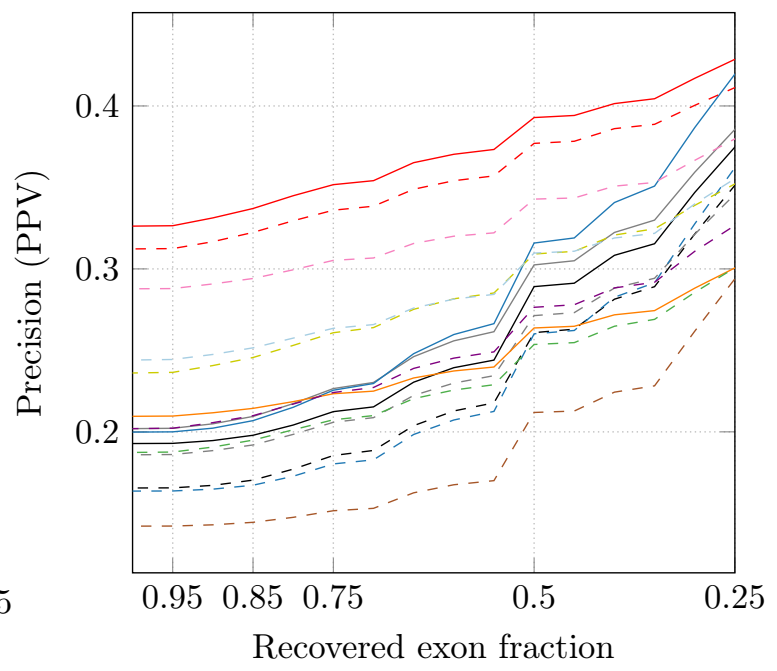

Figure 3: Proportion of reconstructed isoforms classified by SQANTI as FSM or ISM vs. the cumulative proportion of recovered exons from the reference. 


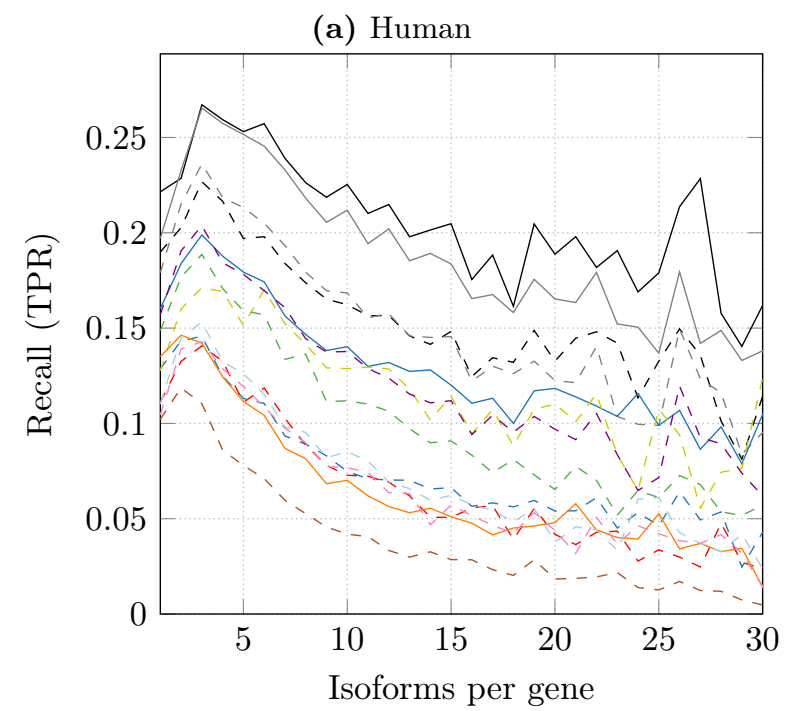

(c) Rice

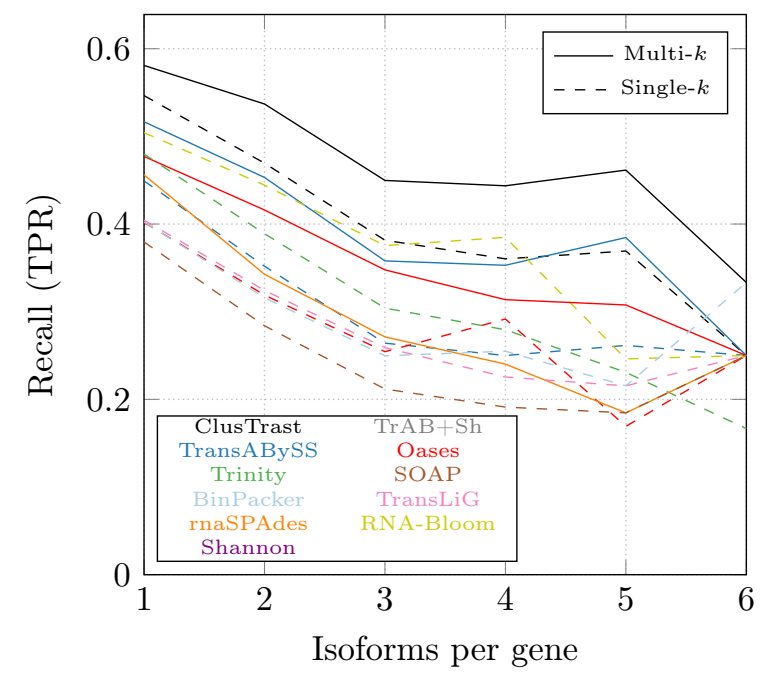

(e) Zebrafish

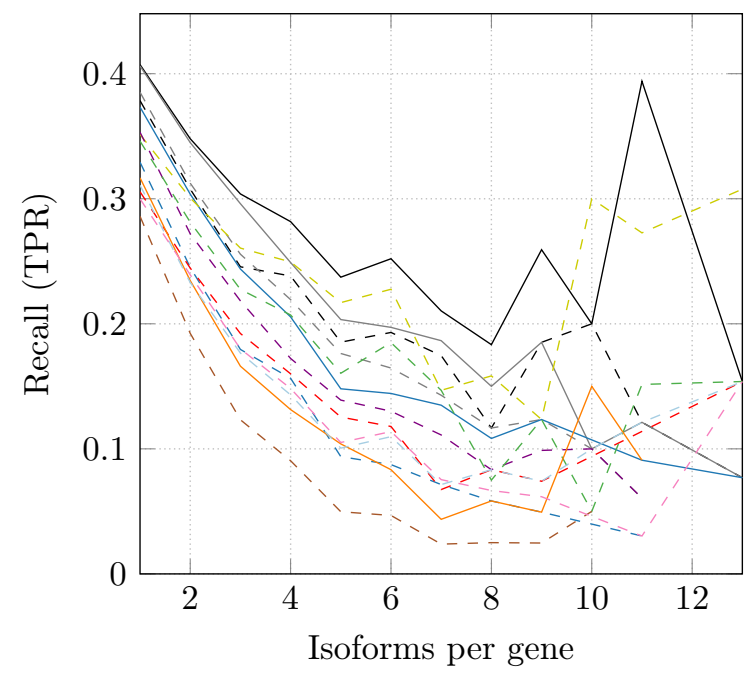

(b) Mouse

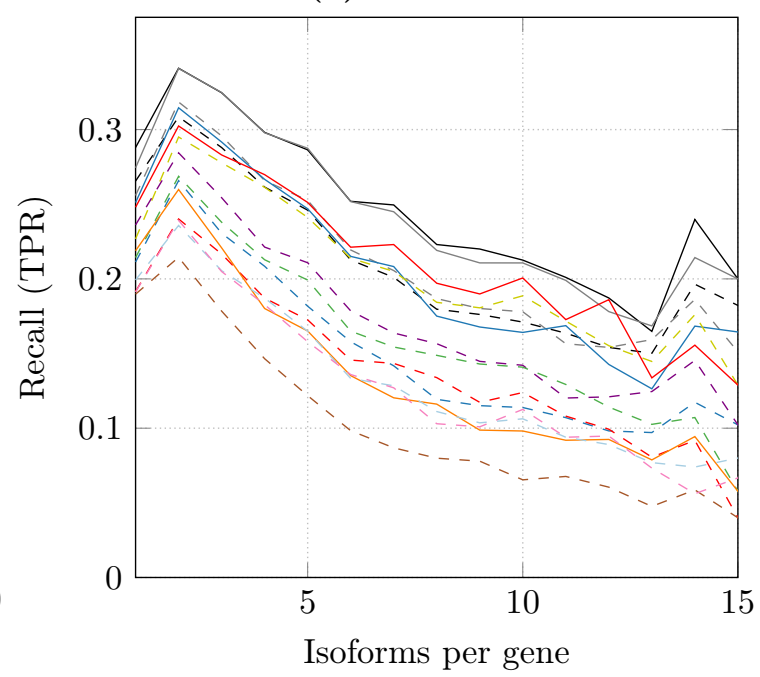

(d) Arabidopsis

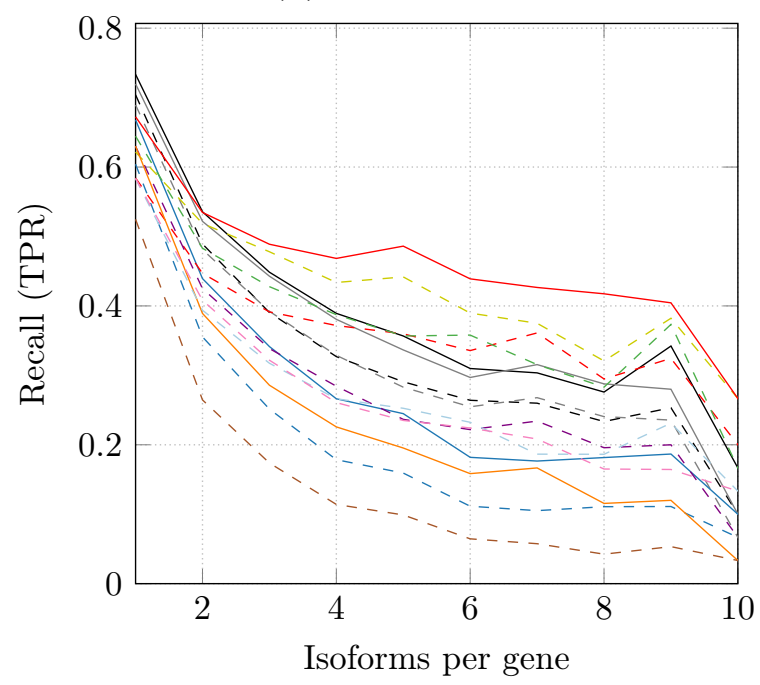

(f) Poplar

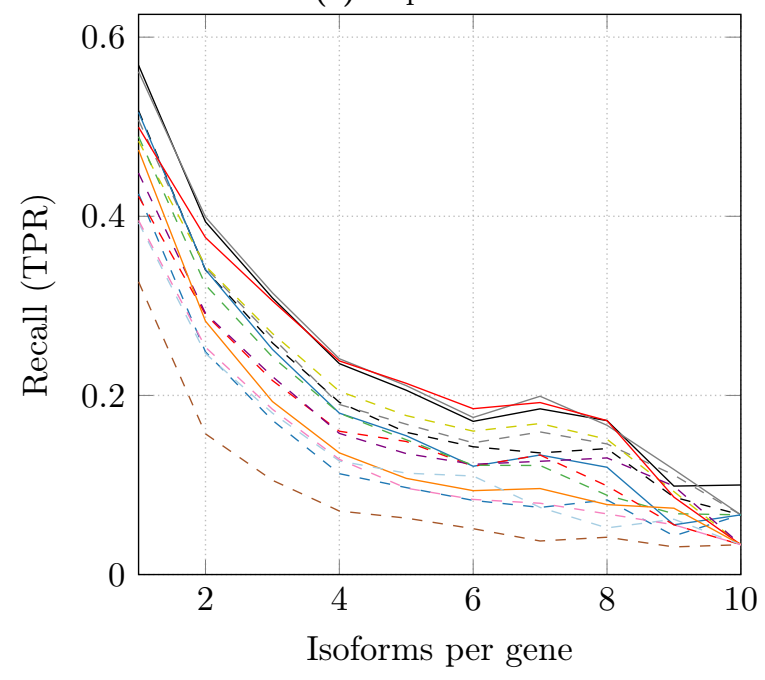

Figure 4: SQANTI recall of reference isoforms (FSM) binned by number of expressed isoforms per gene. 
(a) Human

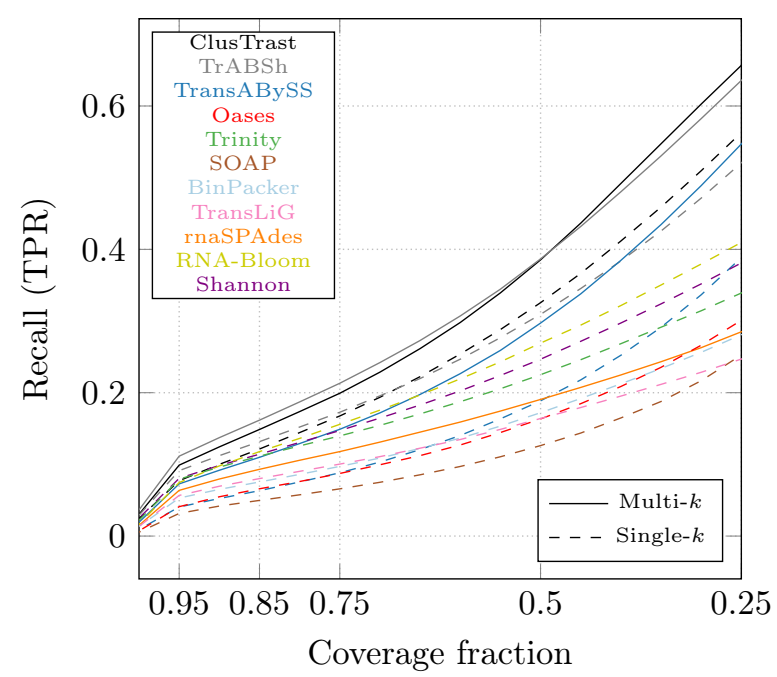

(c) Rice

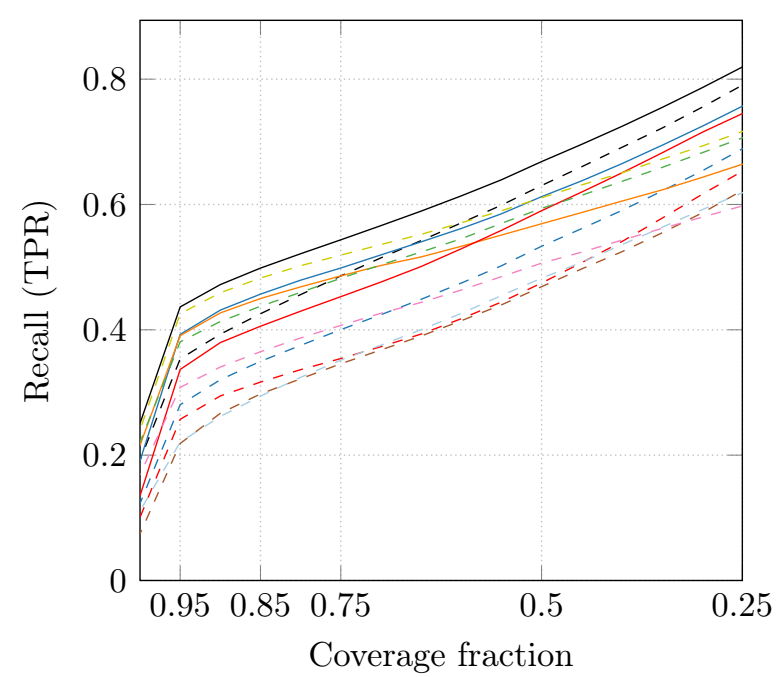

(e) Zebrafish

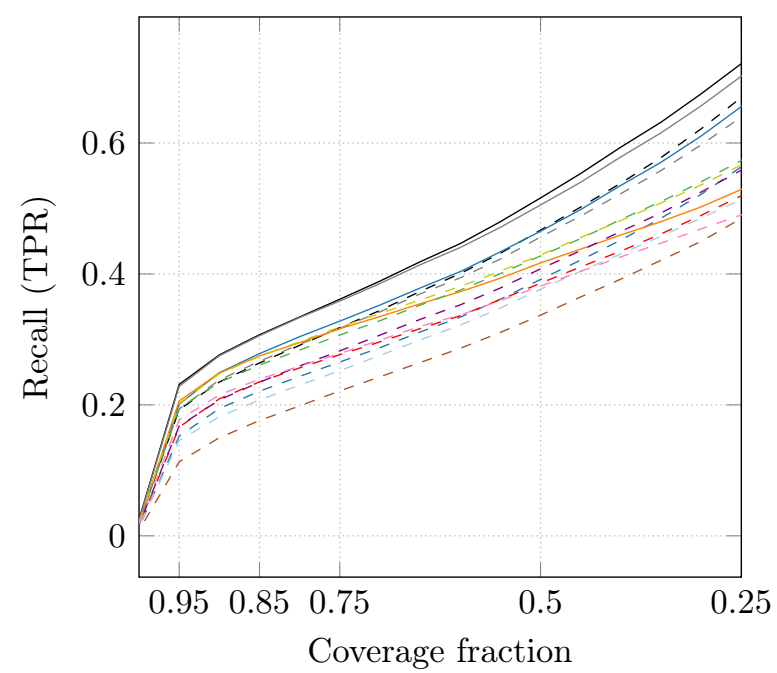

(b) Mouse

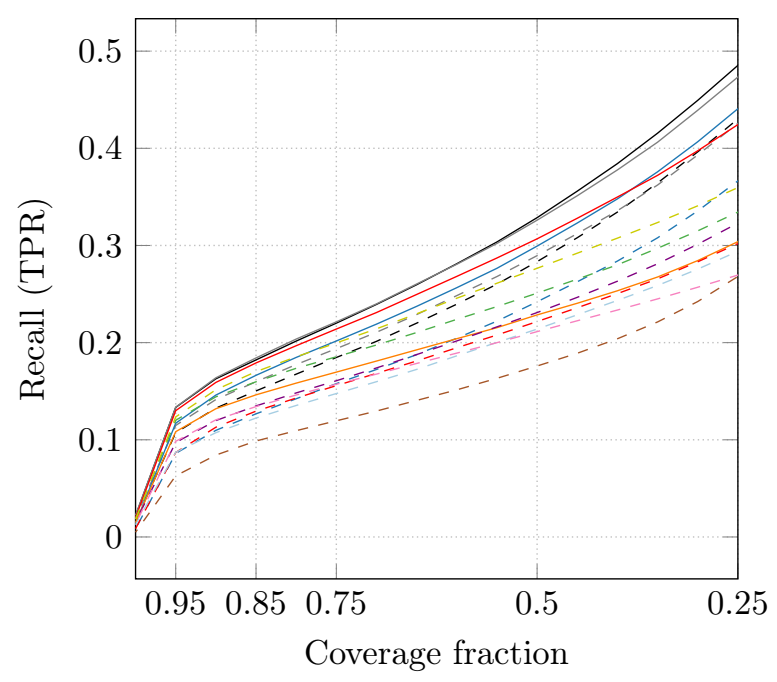

(d) Arabidopsis

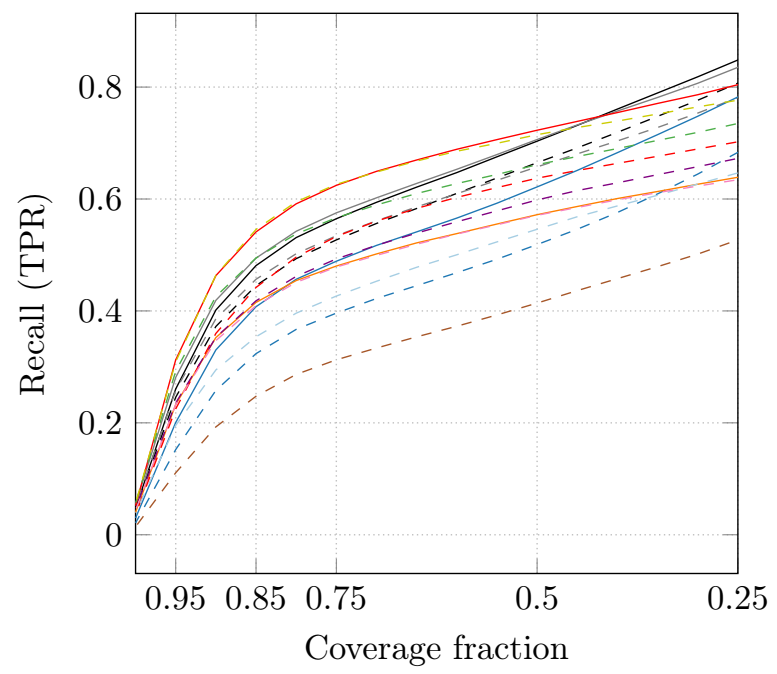

(f) Poplar

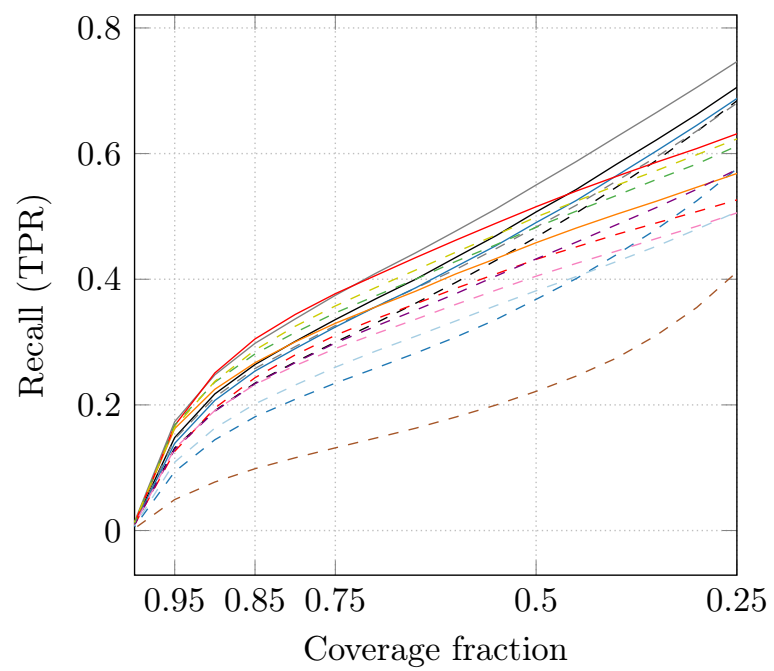

Figure 5: Proportion of references with a CRBB hit vs. the cumulative proportion of recovered reference length. 
(a) Human

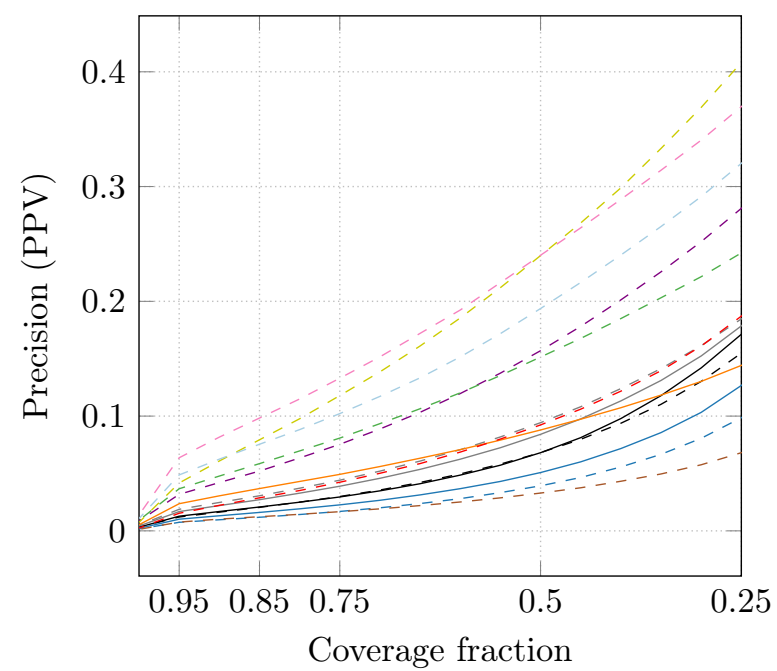

(c) Rice

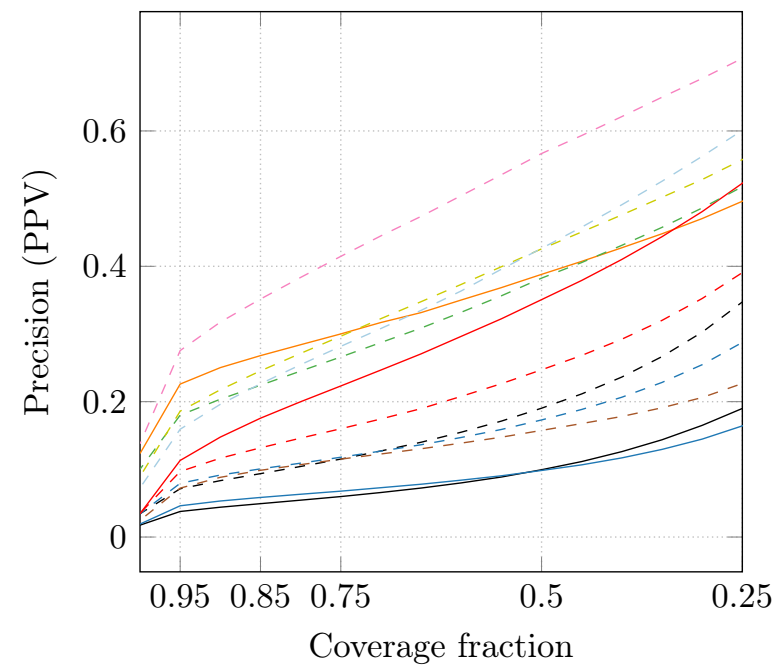

(e) Zebrafish

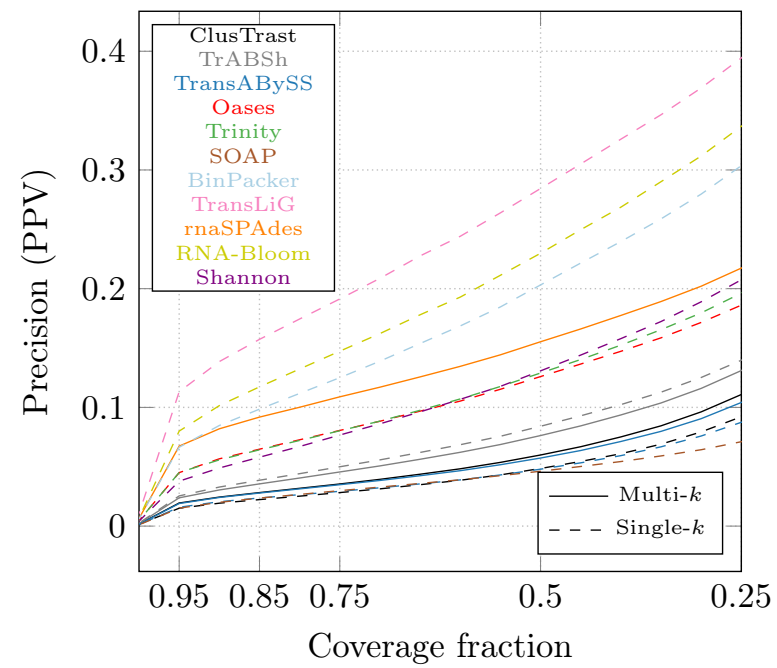

(b) Mouse

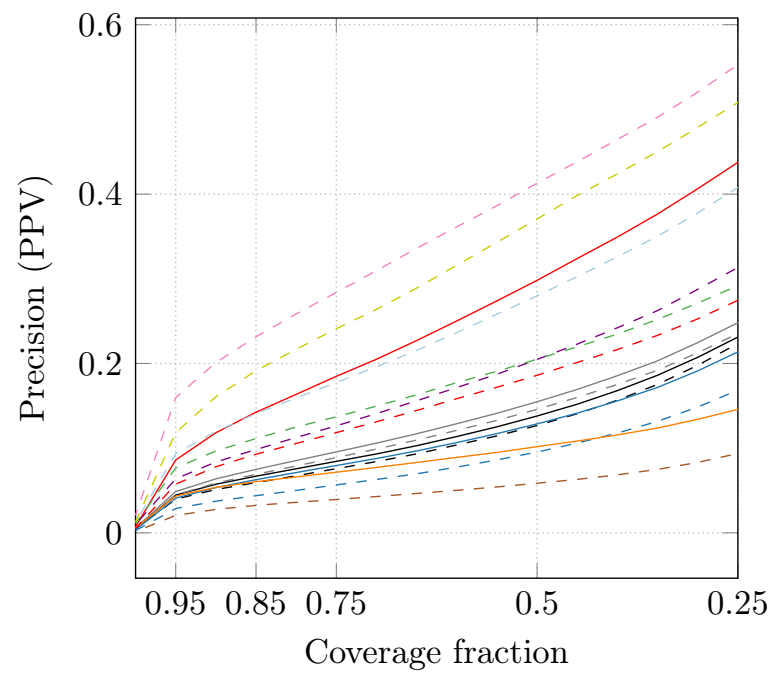

(d) Arabidopsis

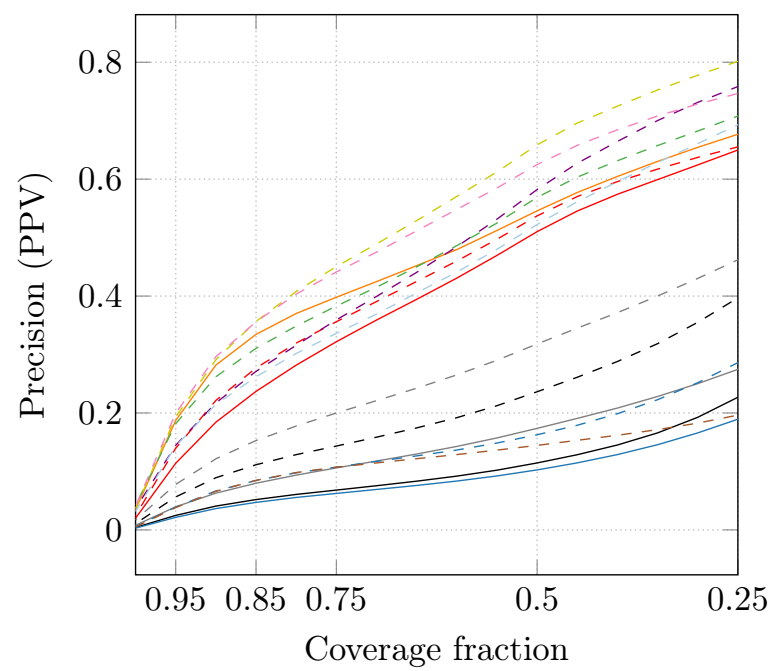

(f) Poplar

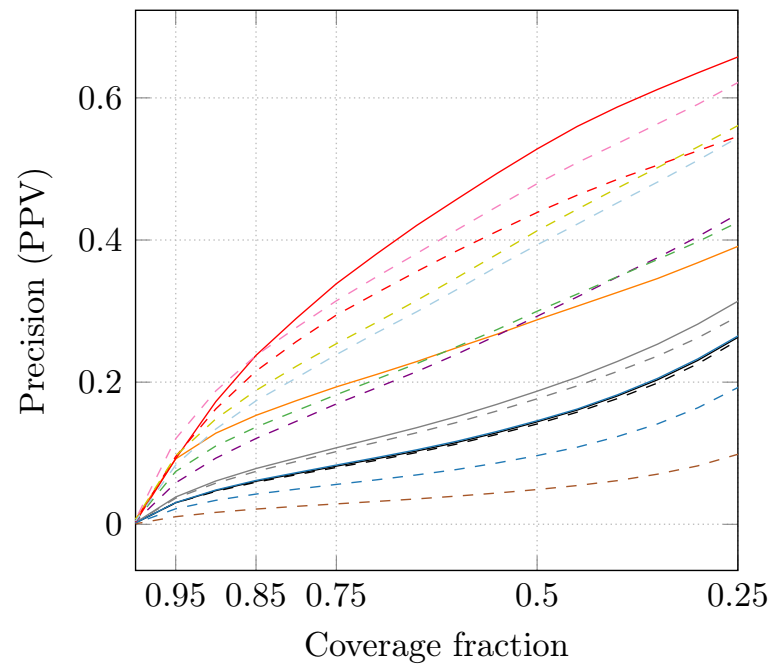

Figure 6: Proportion of reconstructed isoforms with a CRBB hit vs. the cumulative proportion of recovered reference length. 
fold change); the assembler with highest precision in a dataset was always TransLiG, RNABloom, or Oases-M.

\subsubsection{SQANTI and CRBB evaluation metrics are correlated}

SQANTI and CRBB recalls were highly correlated across all assemblies and datasets $(\rho=0.94$; see Supplementary Figure S.5) while SQANTI and CRBB precisions were less correlated $(\rho=$ 0.71; see Supplementary Figure S.6). We observed two modes in the comparison of SQANTI and CRBB precision: one mode has a steeper slope that includes SOAP-denovo-Trans and assemblers based on Trans-ABySS, and the other mode has a shallower slope that includes the other assemblers.

\subsubsection{Reference transcripts are often covered to at least $95 \%$ by FSMs}

We investigated the number of expressed reference transcript isoforms that were reconstructed to at least $50 \%$ and $95 \%$ of their length by a single FSM according to SQANTI, Table S.9. For all assemblies and both length requirements, either $\operatorname{Tr} \mathrm{AB}+\mathrm{Sh}$ or ClusTrast reconstructed the most reference transcript isoforms, with small differences $(<5 \%)$ except for rice where $\mathrm{TrAB}+\mathrm{Sh}$ did not produce a result. Between $47.8 \%$ (arabidopsis) and $68.8 \%$ (rice) of the reference transcript isoforms that had an FSM match were reconstructed by the FSM-classified contig from ClusTrast to at least $95 \%$ of their length. The corresponding range for reconstruction to at least 50\% of the reference transcript length was between $75.6 \%$ (human) and $92.1 \%$ (arabidopsis).

\section{Discussion}

We described and assessed the de novo transcriptome assembler ClusTrast. Across all six tested datasets, ClusTrast-M and ClusTrast-S created assemblies that had the highest or among the highest recall as measured by SQANTI and CRBB.

Comparing ClusTrast-M to one of the most popular transcriptome assemblers, Trinity, revealed that ClusTrast- $\mathbf{M}$ detected more transcript isoforms than Trinity, and also had a higher precision for isoforms as measured by SQANTI, but clearly underperformed according to CRBB precision. The difference in relative performance of ClusTrast and Trinity according to CRBB and SQANTI precision may be explained by how CRBB handles assembled transcripts with high similarity: A CRBB hit has to be the top ranked BLAST hit when aligning assembled transcripts to the reference transcriptome and also when aligning reference transcripts to the assembly. If two or more duplicate or highly similar transcripts exist in the assembly (e.g. two isoforms with the same sequence except for a heterozygous SNP), then only one of these transcripts will be considered a CRBB hit and thus a true positive for precision. CRBB precision therefore does not always capture the possibility that an assembled transcript is an accurate representation of a transcript in the reference transcriptome. SQANTI, in contrast, annotates each transcript independently and therefore calls all assembled transcripts that are similar to a reference transcript as a true positive. We assessed this hypothesis by recalculating SQANTI precision while only counting one transcript match for every reference transcript (Supplementary Figure S.7), and we observed a marked reduction in precision of Trans-ABySS-M and ClusTrast across all assemblies (compare Supplementary Figures S.2 and 
S.7). We conclude that when these assemblers generate a transcript with high similarity to a reference transcript, they also tend to generate multiple similar versions of this transcript.

We observed that ClusTrast generally recovered as many or more known isoforms as TrAB + Sh as measured by SQANTI (Supplementary Figure S.2) while suffering only a small reduction in CRBB precision (Supplementary Figure S.4) and that ClusTrast finished successfully on the rice dataset, where Shannon (and thus $\operatorname{Tr} \mathrm{AB}+\mathrm{Sh}$ ) failed to create an assembly. An explanation for both observations is that the clustering performed in ClusTrast may simplify sub-graphs enough to allow better handling by the Shannon heuristic (Section 3.1.4) and thereby increases sensitivity. ClusTrast-M and TrAB-M+Sh were also the best in reconstructing isoforms to their full length according to SQANTI (Section 3.2.4).

In Figure 4 we compared how many of the expressed isoforms each assembler managed to reconstruct with respect to the number of expressed isoforms originating from the same gene. We observed that ClusTrast- $\mathbf{M}$ and Oases- $\mathbf{M}$ (when it managed to complete) were better than other assemblers at reconstructing isoforms originating from genes expressing multiple isoforms, but both ClusTrast- $\mathbf{M}$ and Oases- $\mathbf{M}$ were highly ranked with respect to recall even without taking number of isoforms per gene into consideration (see Supplementary Figures S.2-S.4).

In our evaluation of ClusTrast and the other transcriptome assemblers, we have emphasized metrics that do not penalize the reconstruction of different isoforms of a gene. A compact transcriptome assembly is, for instance, preferable when the reconstructed transcripts are intended to be used for aligning reads to a "reference" for, e.g., differential gene expression analysis. In such a situation, our approach would not be helpful. However, when the goal is to find as many supported transcript isoforms as possible, compactness is not in itself desirable and could in fact be counter productive. In a recent review by Thind et al. (2021), the authors point out that there is a need for metrics that better capture the performance with regards to transcript isoforms. Our use of SQANTI's approach to evaluation is an attempt to address this. SQANTI (Tardaguila et al., 2018) was originally designed to evaluate long reads (e.g., PacBio CCS) but the categories it defined and its aim to classify each non-redundant transcript individually are useful also in evaluation of assembled transcripts. We have been conservative in that we included only the FSM and ISM categories as true positives. Also the NIC category, which encompasses reconstructed transcripts that contain annotated reference exons only, but in novel combinations, could have been included.

We compared the results from SQANTI and CRBB (which has been used for transcriptome assembly benchmarking before), and as is shown in section 3.2.3, there is a correlation between SQANTI and CRBB scores, particularly strong for recall. This supports the notion that SQANTI is possible to use for transcriptome assembly evaluation.

In our tests of model organisms, ClusTrast consistently detected the most transcript isoforms, but at a cost of lower precision. Therefore, we believe researchers interested in a more complete representation of transcript isoforms from eukaryotic organisms that lack a useful reference genome may wish to use ClusTrast. This includes, e.g., the study of alternative splicing.

\section{Funding}

This work was supported by FORMAS [2013-650] and the Swedish Research Council [201805973]. Computations were enabled by resources, ParallellDatorCentrum (PDC) at KTH Royal 

made available under aCC-BY 4.0 International license.

Institute of Technology, provided by the Swedish National Infrastructure for Computing (SNIC), partially funded by the Swedish Research Council [2018-05973]. We also wish to thank Pelin Akan Sahlén at KTH for access to the server SAGA. 


\section{References}

S. Akhter, W. W. Kretzschmar, V. Nordal, N. Delhomme, N. Street, O. Nilsson, O. Emanuelsson, and J. F. Sundström. Integrative analysis of three RNA sequencing methods identifies mutually exclusive exons of MADS-box isoforms during early bud development in Picea abies. Frontiers in Plant Science, 9:1625, 2018. ISSN 1664-462X. doi: 10.3389/fpls.2018. 01625 .

S. F. Altschul, W. Gish, W. Miller, E. W. Myers, and D. J. Lipman. Basic Local Alignment Search Tool. Journal of Molecular Biology, 215(3):403-410, 1990. ISSN 0022-2836. doi: https://doi.org/10.1016/S0022-2836(05)80360-2. URL https://www. sciencedirect.com/ science/article/pii/S0022283605803602.

S. Aubry, S. Kelly, B. M. C. Kümpers, R. D. Smith-Unna, and J. M. Hibberd. Deep Evolutionary Comparison of Gene Expression Identifies Parallel Recruitment of Trans-Factors in Two Independent Origins of C4 Photosynthesis. PLOS Genetics, 10(6):1-16, 06 2014. doi: 10.1371/journal.pgen.1004365. URL https://doi.org/10.1371/journal.pgen. 1004365.

N. L. Barbosa-Morais, M. Irimia, Q. Pan, H. Y. Xiong, S. Gueroussov, L. J. Lee, V. Slobodeniuc, C. Kutter, S. Watt, R. Colak, T. Kim, C. M. Misquitta-Ali, M. D. Wilson, P. M. Kim, D. T. Odom, B. J. Frey, and B. J. Blencowe. The Evolutionary Landscape of Alternative Splicing in Vertebrate Species. Science, 338(6114):1587-1593, Dec. 2012. ISSN 0036-8075, 1095-9203. doi: 10.1126/science.1230612.

E. Bushmanova, D. Antipov, A. Lapidus, and A. D. Prjibelski. rnaSPAdes: a de novo transcriptome assembler and its application to RNA-Seq data. GigaScience, 8(9), 09 2019. ISSN 2047217X. doi: 10.1093/gigascience/giz100. URL https://doi.org/10.1093/gigascience/ giz100. giz100.

S. Chen, Y. Zhou, Y. Chen, and J. Gu. fastp: an ultra-fast all-in-one FASTQ preprocessor. Bioinformatics, 34(17):i884-i890, 09 2018. ISSN 1367-4803. doi: 10.1093/bioinformatics/ bty560. URL https://doi.org/10.1093/bioinformatics/bty560.

J. D. Fackenthal and L. A. Godley. Aberrant RNA splicing and its functional consequences in cancer cells. Disease Models \& Mechanisms, 1(1):37-42, 2008. ISSN 1754-8403. doi: 10.1242/dmm.000331. URL https://dmm.biologists.org/content/1/1/37.

S. N. Floor and J. A. Doudna. Tunable protein synthesis by transcript isoforms in human cells. eLife, 5:e10921, Jan. 2016. ISSN 2050-084X. doi: 10.7554/eLife.10921.

M. Garber, M. G. Grabherr, M. Guttman, and C. Trapnell. Computational methods for transcriptome annotation and quantification using RNA-seq. Nature Methods, 8(6):469477, Jun 2011. ISSN 1548-7105. doi: 10.1038/nmeth.1613. URL https://doi.org/10. 1038/nmeth. 1613 .

M. G. Grabherr, B. J. Haas, M. Yassour, J. Z. Levin, D. A. Thompson, I. Amit, X. Adiconis, L. Fan, R. Raychowdhury, Q. Zeng, Z. Chen, E. Mauceli, N. Hacohen, A. Gnirke, N. Rhind, F. di Palma, B. W. Birren, C. Nusbaum, K. Lindblad-Toh, N. Friedman, and A. Regev. Full-length transcriptome assembly from RNA-Seq data without a reference genome. Nat. Biotechnol., 29(7):644-52, May 2011. ISSN 1546-1696. doi: 
10.1038/nbt.1883. URL http://www.ncbi.nlm.nih.gov/pubmed/21572440http://www . pubmedcentral.nih.gov/articlerender.fcgi?artid=PMC3571712.

K. E. Hayer, A. Pizarro, N. F. Lahens, J. B. Hogenesch, and G. R. Grant. Benchmark analysis of algorithms for determining and quantifying full-length mRNA splice forms from RNA-seq data. Bioinformatics, page btv488, Sept. 2015. ISSN 1367-4803, 1460-2059. doi: 10.1093/bioinformatics/btv488.

M. Hölzer and M. Marz. De novo transcriptome assembly: A comprehensive cross-species comparison of short-read RNA-Seq assemblers. GigaScience, 8(5), 05 2019. ISSN 2047-217X. doi: 10.1093/gigascience/giz039. URL https://doi.org/10.1093/gigascience/giz039. giz039.

S. Kannan, J. Hui, K. Mazooji, L. Pachter, and D. Tse. Shannon: An Information-Optimal de Novo RNA-Seq Assembler. Preprint at bioRxiv, 2016. URL https://www.biorxiv.org/ content/early/2016/02/09/039230.

B. Li and C. N. Dewey. RSEM: accurate transcript quantification from RNA-Seq data with or without a reference genome. BMC Bioinformatics, 12(1):323, Aug 2011. ISSN 1471-2105. doi: 10.1186/1471-2105-12-323. URL https://doi .org/10.1186/1471-2105-12-323.

H. Li. Minimap2: pairwise alignment for nucleotide sequences. Bioinformatics, 34(18):30943100, 05 2018. ISSN 1367-4803. doi: 10.1093/bioinformatics/bty191. URL https://doi. org/10.1093/bioinformatics/bty191.

J. Liu, G. Li, Z. Chang, T. Yu, B. Liu, R. McMullen, P. Chen, and X. Huang. BinPacker: Packing-based de novo transcriptome assembly from RNA-seq data. PLoS computational biology, 12(2):e1004772-e1004772, Feb 2016. ISSN 1553-7358. doi: 10.1371/journal.pcbi. 1004772. URL https://www.ncbi.nlm.nih.gov/pubmed/26894997.

J. Liu, T. Yu, Z. Mu, and G. Li. TransLiG: a de novo transcriptome assembler that uses line graph iteration. Genome Biology, 20(1):81, 2019. ISSN 1474-760X. doi: 10.1186/ s13059-019-1690-7. URL https://doi.org/10.1186/s13059-019-1690-7.

K. M. Nip, R. Chiu, C. Yang, J. Chu, H. Mohamadi, R. L. Warren, and I. Birol. RNABloom enables reference-free and reference-guided sequence assembly for single-cell transcriptomes. Genome Research, 30(8):1191-1200, 2020. doi: 10.1101/gr.260174.119. URL http://genome.cshlp.org/content/30/8/1191. abstract.

G. Robertson, J. Schein, R. Chiu, R. Corbett, M. Field, S. D. Jackman, K. Mungall, S. Lee, H. M. Okada, J. Q. Qian, M. Griffith, A. Raymond, N. Thiessen, T. Cezard, Y. S. Butterfield, R. Newsome, S. K. Chan, R. She, R. Varhol, B. Kamoh, A.-L. Prabhu, A. Tam, Y. Zhao, R. A. Moore, M. Hirst, M. A. Marra, S. J. M. Jones, P. A. Hoodless, and I. Birol. De novo assembly and analysis of RNA-seq data. Nature Methods, 7:909-912, Oct 2010. URL https://doi.org/10.1038/nmeth.1517.

K. Sahlin and P. Medvedev. De novo clustering of long-read transcriptome data using a greedy, quality value-based algorithm. Journal of Computational Biology, 27(4):472-484, 2020. doi: 10.1089/cmb.2019.0299. URL https://doi.org/10.1089/cmb.2019.0299. PMID: 32181688 . 
M. H. Schulz, D. R. Zerbino, M. Vingron, and E. Birney. Oases: Robust de novo RNA-seq assembly across the dynamic range of expression levels. Bioinformatics, 28(8):1086-1092, 2012. ISSN 13674803. doi: 10.1093/bioinformatics/bts094.

R. Smith-Unna, C. Boursnell, R. Patro, J. M. Hibberd, and S. Kelly. TransRate: reference-free quality assessment of de novo transcriptome assemblies. Genome Research, 26(8):1134-1144, 2016. doi: 10.1101/gr.196469.115. URL http://genome.cshlp.org/content/26/8/1134. abstract.

T. Sterne-Weiler and J. R. Sanford. Exon identity crisis: Disease-causing mutations that disrupt the splicing code. Genome Biology, 15(1):201, 2014. ISSN 1465-6906. doi: 10.1186/ gb4150.

M. Tardaguila, L. de la Fuente, C. Marti, C. Pereira, F. J. Pardo-Palacios, H. del Risco, M. Ferrell, M. Mellado, M. Macchietto, K. Verheggen, M. Edelmann, I. Ezkurdia, J. Vazquez, M. Tress, A. Mortazavi, L. Martens, S. Rodriguez-Navarro, V. Moreno, and A. Conesa. SQANTI: extensive characterization of long read transcript sequences for quality control in full-length transcriptome identification and quantification. Genome Research, 28(1):1-16, 2 2018. doi: 10.1101/gr.222976.117. URL http://genome.cshlp.org/content/early/2018/ 02/09/gr.222976.117.abstract.

A. S. Thind, I. Monga, P. K. Thakur, P. Kumari, K. Dindhoria, M. Krzak, M. Ranson, and B. Ashford. Demystifying emerging bulk RNA-Seq applications: the application and utility of bioinformatic methodology. Briefings in Bioinformatics, 22(6), 07 2021. ISSN 1477-4054. doi: 10.1093/bib/bbab259. URL https://doi.org/10.1093/bib/bbab259. bbab259.

E. T. Wang, R. Sandberg, S. Luo, I. Khrebtukova, L. Zhang, C. Mayr, S. F. Kingsmore, G. P. Schroth, and C. B. Burge. Alternative isoform regulation in human tissue transcriptomes. Nature, 456(7221):470-476, Nov. 2008. ISSN 0028-0836, 1476-4687. doi: 10.1038/nature07509.

Y. Xie, G. Wu, J. Tang, R. Luo, J. Patterson, S. Liu, W. Huang, G. He, S. Gu, S. Li, X. Zhou, T.-W. Lam, Y. Li, X. Xu, G. K.-S. Wong, and J. Wang. SOAPdenovo-Trans: de novo transcriptome assembly with short RNA-Seq reads. Bioinformatics, 30(12):1660-1666, 02 2014. ISSN 1367-4803. doi: 10.1093/bioinformatics/btu077. URL https://doi.org/10. 1093/bioinformatics/btu077.

H. Y. Xiong, B. Alipanahi, L. J. Lee, H. Bretschneider, D. Merico, R. K. C. Yuen, Y. Hua, S. Gueroussov, H. S. Najafabadi, T. R. Hughes, Q. Morris, Y. Barash, A. R. Krainer, N. Jojic, S. W. Scherer, B. J. Blencowe, and B. J. Frey. The human splicing code reveals new insights into the genetic determinants of disease. Science, 347(6218):1254806, Jan. 2015. ISSN 0036-8075, 1095-9203. doi: 10.1126/science.1254806. URL https://www.science. org/doi/abs/10.1126/science.1254806. 